\title{
Notas taxonômicas em Verbenaceae do Brasil
}

\author{
Taxonomic notes in Verbenaceae of Brazil
}

\author{
Fátima Regina G. Salimena ${ }^{1,3}$ \& Maria Ema Múlgura ${ }^{2}$
}

\begin{abstract}
Resumo
Como parte da revisão da família Verbenaceae para a Flora do Brasil são apresentados dois sinônimos novos, e uma combinação nova no gênero Lantana, bem como um nome novo, dez sinônimos novos e dez lectotipificações no gênero Lippia e dois sinônimos novos em Stachytarpheta.

Palavras-chave: Flora do Brasil, Lantana, Lippia, Stachytarpheta,taxonomia.
\end{abstract}

\begin{abstract}
As part of the revision of the family Verbenaceae for the Flora of Brazil, two new synonyms and one new combination in the genus Lantana, as well as one new name, ten new synonyms and ten lectotypifications in the genus Lippia and two new synonyms in Stachytarpheta are presented.
\end{abstract}

Key words: Flora of Brazil, Lantana, Lippia, Stachytarpheta, taxonomy.

\section{Introdução}

Verbenaceae (senso Cantino et al.1992) reúne cerca de 32 gêneros e 480 espécies, com distribuição neotropical (Atkins 2004). O Brasil reúne a maior riqueza da família, com 16 gêneros e 290 espécies, sendo 191 endêmicas (Salimena et al. 2014). Os gêneros mais representativos na flora brasileira são Lippia L., com 88 espécies, sendo 68 endêmicas (Salimena \& Múlgura 2014), e Stachytarpheta Vahl, representado por 81 espécies, 75 endêmicas (Salimena 2014), ambos com maior riqueza nos cerrados e campos rupestres do Planalto Central e Cadeia do Espinhaço. Lantana L. está representado por 22 espécies na flora brasileira, das quais 12 são endêmicas (Silva \& Salimena 2014).

Stachytarpheta está incluído na tribo Duranteae Benth., que reúne os gêneros com inflorescências arranjadas em espigas terminais ou racemos compostos, com ramos floríferos terminais ou axilares, destacando-se dos demais pela presença de dois estames funcionais e dois estaminódios.

Lantana e Lippia estão incluídos na tribo Lantaneae Endl., que representa cerca de $80 \%$ das espécies de Verbenaceae (Marx et al. 2010). Esta tribo reúne os gêneros com cálice persistente no fruto, corola 4-5 lobada e ovário unicarpelar, que se desenvolve em drupa 2-locular com pireno 2-seminado em Lantana ou esquizocarpo, separando-se em dois mericarpos uniseminados na maturidade, em Lippia (Atkins 2004). A morfologia do fruto tem sido o caráter classicamente utilizado na delimitação destes gêneros. Apesar da importância na taxonomia e delimitação de ambos os gêneros, este caráter é omitido na maioria das descrições originais das espécies destes gêneros, sendo motivo de confusões taxonômicas, necessitando de revisão das coleções das espécies, especialmente as coleções-tipo, para a correta identificação e delimitação de Lantana e Lippia.

Este trabalho é parte de uma ampla revisão e atualização da nomenclatura dos gêneros Lantana, Lippia e Stachytarpheta para o tratamento da família Verbenaceae na Lista de Espécies da Flora do Brasil. Tem como objetivo a publicação das alterações que já estão sendo utilizadas nesta base de dados, e sua correta utilização nas diversas floras em elaboração no país.

\footnotetext{
${ }^{1}$ Universidade Federal de Juiz de Fora, Inst. Ciências Biológicas, Depto. Botânica, 36330-900, Juiz de Fora, Minas Gerais, MG, Brasil.

${ }^{2}$ Instituto de Botánica Darwinion, Labardén 200, CC22, San Isidro, Buenos Aires, Argentina.

${ }^{3}$ Autor para correspondência: fatima.salimena@ufjf.edu.br
} 


\section{Material e Métodos}

Para a revisão e atualização taxonômica de Verbenaceae para a Flora do Brasil foram examinadas as obras prínceps de todas as espécies de Lantana, Lippia e Stachytarpheta com registro para a flora brasileira, bem como examinadas as coleções-tipo destas espécies depositadas nos herbários B, BR, G, NY, K, P, SI, TEX/LL e W (acrônimos segundo Thiers 2014). Além destas, foram examinadas as coleções de Verbenaceae dos seguintes herbários brasileiros: BHCB, CEN, CESJ, HBRC, HUEFS, MBM, RB, SPF, UB e UFG, para complementação dos dados dos táxons.

\section{Resultados}

1. Lantana gracilis T. Silva, Darwiniana 40: 59 . 2002. Lippia bahiensis Moldenke, Phytologia 31(2): 229. 1935, non Lantana bahiensis Turcz., Bull. Soc. Imp. Naturalistes Moscou 36(2): 206.1863. Tipo: BRASIL. Bahia: $23 \mathrm{~km}$ sul de Aracatu, entre Vitória da Conquista e Brumado, 13.I.1974, R.M.Harley, S.A.Renvoize, C.M.Erskine, C.A. Brighton \& R. Pinheiro 15027 (holótipo, TEX/ $\mathrm{LL}$ !; isótipo $\mathrm{K}$ !).

Lippia harleyi Moldenke, Phytologia 31(2): 231. 1975. Tipo. BRASIL. Bahia: Serra do Curral Feio, estrada para Minas do Mimoso, $8 \mathrm{~km}$ nordeste de Lagoinha, 5,5 km sudeste de Delfino, $10^{\circ} 24^{\prime} \mathrm{S}$ $041^{\circ} 17^{\prime}$ 'W, 5.III.1974, R.M. Harley, S.A. Renvoize, C.M. Erskine, C.A. Brighton \& R. Pinheiro 16788 (holótipo, TEX/LL!; isótipo MO!), syn. nov.

Lantana gracilis foi descrita como Lippia bahiensis, sem menção ao fruto, sendo esta característica utilizada por Silva \& Salimena (2002) para a proposição da nova combinação para o gênero Lantana.

O novo sinônimo aqui proposto (Lippia harleyi Moldenke), foi descrito como Lippia, mas a coleção-tipo e outros materiais desta espécie examinados em diferentes coleções de herbários, mostraram que o fruto é uma drupa com um pireno. Além do que as descrições originais de ambas as espécies são praticamente idênticas. Desta forma é apresentado um novo sinônimo para Lantana gracilis T. Silva, espécie endêmica dos campos rupestres do estado da Bahia.

2. Lantana hypoleuca Briq., Bull. Herb. Boissier, sér. 2, 4: 1064. 1904. Tipo: PARAGUAI: "In regione cursus superioris fluminis Apa", 1902, E. Hassler 7778 (lectótipo, G!, designado por T.Silva 2001).
Lippia vinosa Moldenke, Phytologia 28(3): 303. 1974. Tipo: BRASIL: Mato Grosso. Terenos 13.VIII.1970, G. Hatschbach \& O. Guimarães 24621 (holótipo, TEX/LL!; isótipo, MBM!), syn. nov.

Lippia vinosa foi descrita sem as características do fruto que separam Lantana e Lippia. As características morfológicas desta espécie são idênticas às descritas para Lantana hypoleuca, incluindo a filotaxia ternada, lâmina foliar cartácea, discolor, com comprimento de 4-6 $\mathrm{cm}, 2$ inflorescências por nó, menores que as folhas adjacentes, pedúnculos da inflorescência até $2 \mathrm{~cm}$ compr. e brácteas ovais. $\mathrm{O}$ fruto em Lippia vinosa é vináceo (in sched.), caráter que provavelmente lhe conferiu este nome, com exocarpo estriado, idêntico ao apresentado por Lantana hypoleuca. Dessa forma, é proposta a nova sinonímia, expandindo a distribuição geográfica de Lantana hypoleuca, com registros de ocorrência para ao Paraguai e Brasil, nos estados de Goiás, Minas Gerais, São Paulo, Rio de Janeiro e Distrito Federal (Silva \& Salimena 2014) incluindo também o estado do Mato Grosso.

3. Lantana ramboi (Moldenke) Salimena \& Múlgura, comb. nov. Lippia ramboi Moldenke, Lloydia 13(3): 223. 1950. Tipo: BRASIL. Rio Grande do Sul: São Francisco de Paula, 13.III.1950, B. Rambo 46306 (holótipo, NY!).

$\mathrm{Na}$ descrição original de Lippia ramboi não são apresentadas características do fruto. Entretanto, a análise de diversos outros materiais desta espécie, depositados em diferentes herbários, deixa clara a morfologia do fruto, do tipo drupa, característica de Lantana, sendo proposta a nova combinação. Lantana ramboi é caracterizada pelas lâminas foliares obovadas-elípticas e flores amarelas, ocorrendo em bordas de florestas na região Sul do Brasil.

4. Lippia acutidens Mart. \& Schauer in DC., Prodr. 11: 590. 1847. Tipo: BRASIL. "In campis prov. Minarum ad praedium Yha, in confinibus prov. Goyacensis". C.F.V.Martius s.n. (holótipo, M!; isótipo, SI!).

Lippia reticulata Hayek, Repert. Spec. Nov. Regni Veg. 19:87.1906. Tipo: BRASIL. Piauí, G. Gardner 2940 (lectótipo P!, aqui designado; isolectótipos, G!, W!). syn.nov.

Lippia reticulata foi descrita baseada em um síntipo, formado por duas coleções de Gardner no 
Brasil, dos estados do Piauí e Pernambuco, ambas com o mesmo número de coleta (2940). Estas coleções foram examinadas nos herbários $\mathrm{G}, \mathrm{P}$ e $\mathrm{W}$, sendo indicada para lectotipificar a espécie a coleção com procedência do Piauí, depositada em $\mathrm{P}$, por reunir ótimas características do táxon e apresentar excelente estado de conservação.

A análise das coleções-tipo de $L$. reticulata Hayek deixa evidente a sinonímia sob L. acutidens Mart. \& Schauer, espécie bastante característica pelas lâminas foliares coriáceas, ovais, com margem denteada-ciliada. Esta espécie ocorre nos estados da Bahia, Goiás, Maranhão e Tocantins e a referência para Minas Gerais, na coleção de Martius $s / n$, é duvidosa, uma vez que não foram encontrados registros da espécie neste estado. A coleção Gardner 2940, do estado de Piauí, deve da mesma forma referir-se ao atual estado do Maranhão, já que não existe nenhum outro registro da espécie para o Piauí.

5. Lippia alba (Mill.) N.E.Br. ex Britton \& P. Wilson, Bot. Porto Rico 6: 141.1925. Lantana alba J. F. Miller, Gard. Dict. Edit. 8. 1768. Camara alba (Mill.) Kuntze, Rev. Gen. Pl. 2: 504. 1891. Tipo: México. Campeche, W. Houstoun (holótipo, BM!; fotografia, SI).

Zappania odoratissima Scopoli, Deliciae florae et faune insubricae 1: 34. 1786. Lectótipo aqui designado: Deliciae florae et faune insubricae 1 : tab. 15. 1786.

Lippia citrata Willd. ex Cham., Linnaea 7: 214. 1832. Tipo: BRASIL. F.W. Sieber s.n. (lectótipo, HAL 106957!, aqui designado; isótipos, BR!, HAL!).

Lippia globiflora var. normalis Kuntze f. lilacina Kuntze, Revis. Gen. Pl. 3(2): 251. 1898. Tipo: BRASIL. Mato Grosso, VII.1892, C.E.O.Kuntze s.n. (lectótipo, NY137764!, aqui designado).

Lippia alba é uma espécie de ampla distribuição ocorrendo nas Américas, desde o sul dos Estados Unidos, México e Índias Ocidentais até a Argentina e Uruguai, nos mais variados ambientes, ocorrendo em florestas, campos, beira de estradas, sendo muito cultivada para uso medicinal. No Brasil, é frequente na região Amazônica, onde ocorre em bancos de areia ao longo dos rios. Esta ampla distribuição geográfica resulta em grande plasticidade fenotípica, e na descrição de muitos táxons relacionados às variações encontradas nas folhas, incluindo principalmente características relacionadas à forma da lâmina e indumento.
Zappania odoratissima é um nome validamente publicado com uma ilustração e considerada sinônimo de L. alba na Flora do Panamá (Moldenke 1973). Não existem informações sobre coleções de I.A. Scopoli, que trabalhou no Horto Botânico de Pavia, Itália, sendo, portanto, a ilustração designada para tipificar esta espécie.

Lippia globiflora var. normalis Kuntze f. lilacina Kuntze está representada na coleção do herbário NY por três coleções identificadas como “cotipos", por Moldenke. Para lectotipificar este nome foi indicada a coleção NY 137764, que inclui três ramos com flores, bem desenvolvidos, que melhor representa a espécie. Esta coleção inclui uma etiqueta manuscrita com vários sinônimos, outras duas etiquetas manuscritas por C.O.Kuntze, em que nomeia a nova forma lilacina, e outra com os seguintes dados: "Brasilia, 7,1892. Mato Grosso, Playa am Rio Paraguay". A coleção de número NY137765 com estes mesmos dados, inclui dois ramos, mas não inclui a etiqueta de sinônimos. A coleção NY173366 não traz informações sobre a data de coleta e indica apenas a localidade "Concepción de Paraguay".

6. Lippia alnifolia Schauer in DC., Prodr. 11: 588. 1847. Tipo: BRASIL. "Habitat in campis graminosis ad Mumbucas, deserti Min. Nov. Provinciae M. Geraes" C.F.P.Martius s.n. (lectótipo, M!, aqui designado; isótipo, SI!).

Lippia rotundifolia var. bahiensis Moldenke, Phytologia 48: 290. 1980. Tipo: BRASIL. Bahia, Palmeiras, 31.X. 1979, S .Mori 12919 (holótipo, TEX!), syn.nov.

Lippia alnifolia foi descrita baseada na coleção de Martius s.n., havendo no protólogo a referência: "In campis graminosis Brasiliae, in Prov. Minarum et Bahiensis". Para a lectotipificação desta espécie, foi designada a coleção de Martius s.n, depositada em M, procedente de Minas Gerais, que apresenta todas as caraterísticas da espécie. Entretanto, a procedência desta coleção deve ser considerada para os limites geográficos estabelecidos para a época, uma vez que não há nenhum registro de L. alnifolia até o momento para o estado de Minas Gerais, sendo a espécie endêmica do estado da Bahia.

7. Lippia aristata Schauer, in DC Prodr. 11: 581. 1847. Lantana aristata (Schauer) Briq., Archiv. Bot. 2(10): 17. 1904. Tipo: BRASIL. Goiás: 1839, G. Gardner 3403 (lectótipo, K!, designado por Silva 2001; isolectótipos, BM!, K!, SI!). 
Lantana aristata var. hoehnei Moldenke, Phytologia 3: 112. 1949. Tipo: Brasil. Mato Grosso: Coxipó da Ponte, F. C. Hoehne, Com. Rondon 2821(holótipo, $\mathrm{NY}$ !), syn. nov.

Lantana aristata var. hoehnei foi descrita com base na forma das brácteas, que diferem da variedade tipo pelo ápice obtuso. A análise do holótipo desta variedade deixa clara sua identificação como Lippia com base no tipo de fruto, enquanto a morfologia das brácteas não apresenta diferenças que sustentem a variedade, sendo portanto proposta a sua sinonímia.

8. Lippia felippei Moldenke, Phytologia 10(3): 171. 1964. Tipo: Brasil. Minas Gerais: Prata, 20.III.1963, G.M.Felippe 140 (holótipo, US!; isótipos, HB!, HUFU!, NY!, RB!, SP!).

Lippia lupulina var. paraguariensis Chodat, Bull. Herb. Boissier, ser. 2, 2: 820. 1902. Tipo: Paraguai: "In campo Apépu (Tapiraguay)", VIII, E. Hassler 4335 (lectótipo NY! designado por Salimena 2002: 12, isótipo P!), syn. nov.

Lippia felippei é caracterizada pelas lâminas foliares cordiformes e reflexas, além de brácteas membranáceas róseas. Ocorre no Paraguai e no Brasil, na região do cerrado nas divisas dos estados de Minas Gerais e São Paulo.

Lippia lupulina var. paraguariensis Chodat foi considerada um sinônimo de Lippia renifolia Turcz. (Salimena 2002). Entretanto, após o exame das coleções-tipo desses nomes, tornou-se evidente que não são sinônimos. As características da lâmina foliar cordiforme, com base cordada, margem denteada e posição reflexa da folha no caule, permitem separar Lippia lupulina var. paraguariensis de Lippia lupulina Cham., sendo a variedade proposta por Chodat (1902) claramente um sinônimo de L. felippei, que apresenta estas mesmas características diagnósticas.

9. Lippia filifolia Mart. \& Schauer, in DC. Prodr. 11: 586. 1847. Tipo: BRASIL. Minas Gerais: Serra de Santo Antônio, C.F.P. Martius s.n (lectótipo, M 110863!, aqui designado)

Lippia linearifolia Moldenke, Phytologia 24: 454. 1972. Tipo: BRASIL. Minas Gerais: Gouveia, Serra do Espinhaço, 12.XI.1971, G. Hatschbach 27812 (holótipo, TEX!; isótipos, MBM!, MO!), syn. nov.

Lippia linearifolia apresenta como características principais as lâminas muito estreitas, filiformes e com indumento glanduloso-viscoso, além de flores amarelas. Estas são também as características descritas anteriormente para Lippia filifolia. A análise das coleções-tipo de ambas as espécies, permitiu reconhecer que $L$. filifolia e $L$. linearifolia correspondem a um único táxon e que o primeiro nome tem prioridade, sob o ponto de vista nomenclatural, razão pela qual $L$. linearifolia é sinonimizado. Na descrição original de L. filifolia são citadas duas coleções de Martius e Riedel para Minas Gerais. Foi designada a coleção Martius s.n, depositada no herbário M, com registro 110863 , para lectotipificar L. filifolia por melhor representar as características desta espécie.

10. Lippia ganevii Salimena \& Múlgura, nom. nov. Lantana hatschbachii Moldenke (1977:164), non Lippia hatschbachii Moldenke. Tipo: BRASIL: Bahia. Morro do Chapéu, 15.I.1977, G. Hatschbach 39651 (holótipo, TEX!; isótipo, MBM!).

Lantana hatschbachii foi descrita por Moldenke (1977) sem mencionar as características do fruto. Como este caráter é a base para delimitação dos gêneros Lantana e Lippia, a análise de diversas coleções de herbários desta espécie, provenientes do estado da Bahia e que se encontravam sem identificação, principalmente nos herbários HUEFS, K e SPF, mostraram que o fruto é seco do tipo esquizocárpico, tratando-se portanto de uma espécie de Lippia e desta forma, necessária a sua transferência para este gênero. Como o epíteto "hatschbachii" encontra-se pré-ocupado em Lippia, foi proposto o nome novo Lippia ganevii para substituí-lo em homenagem às ricas coleções desta espécie feitas por Wilson Ganevi, que serviram de base para esta sinonimização.

11. Lippia grandiflora Mart. \& Schauer, in DC Prodr. 11: 591. 1847. Tipo: BRASIL. Minas Gerais, C.F.P.Martius 1571 (holótipo, M!; isótipo TEX/ LL!).

Lantana glaziovii Moldenke, Phytologia 1: 462. 1940. Tipo: BRASIL. Goiás: Près de Ponta Alta, 26.VII.1894, A.F.M.Glaziou 21892 (holótipo, G!; isótipos B!, NY!, P!, SI!), syn. nov.

Lippia grandiflora foi descrita com base em uma coleção de Martius, procedente de Minas Gerais. Moldenke (1965) cita como coleçãotipo Martius 157, mas o número correto desta coleção é Martius 1571. Esta espécie se destaca pelo hábito cespitoso e reduzido, ocorrendo em áreas campestres no Brasil, Paraguai e nordeste da Argentina, especialmente nos cerrados, com floração pós-queimadas. Lantana glaziovii 
Moldenke apresenta as mesmas características morfológicas de Lippia grandiflora, incluindo o hábito reduzido, sistema subterrâneo desenvolvido, e a análise da coleção-tipo indica que o fruto é do tipo esquizocárpico, característica ausente em ambas as descrições originais, sendo proposta a sinonimização de Lantana glaziovii.

12. Lippia lacunosa Mart. \& Schauer in DC., Prodr. 11: 590. 1847. Tipo: BRASIL. Minas Gerais, J.B.E.Pohl 131(lectótipo, K!, aqui designado; isolectótipos, $\mathrm{M}$ !, P!, SI!, W!).

Lippia rotundifolia var. cordata Moldenke, Phytologia 52(6): 414. 1983. Tipo. BRASIL. Distrito Federal, Estação Florestal Cabeça de Veado, 27.VIII.1975, E.P.Heringer 14827 (holótipo, NY!), syn.nov.

Lippia rotundifolia var. cordata foi descrita por Moldenke (1983), que reconheceu como caráter diagnóstico desta variedade a base da lâmina foliar cordada. A análise do holótipo desta variedade além de diversas coleções procedentes do Distrito Federal, indicam que esta variedade é um sinônimo de Lippia lacunosa, sendo todas as demais características morfológicas idênticas à esta espécie, sendo portanto um novo sinônimo.

13. Lippia lasiocalycina Cham., Linnaea 7: 231. 1832. Tipo: BRASIL: F.Sellow B 1452 (lectótipo, K!, aqui designado; foto, Field Museum!, MO!) Lippia subracemosa Mansf., Notizbl. Bot. Garden Berlin 9: 155. 1924. Tipo. BRASIL. Bahia, S. Maria, Pao de Moco, 1913, P.V.Luetzelburg 710 (lectótipo M!, aqui designado; foto K!, foto Field Museum 17549!) syn. nov.

O holótipo de Lippia lasiocalycina, representado pela coleção Sellow s.n., depositado no herbário de Berlim (B), sem localidade e sem data, foi destruído durante a Segunda Guerra Mundial, sem informações adicionais no protólogo. Esta coleção foi fotografada por Macbride sob o número 17522, depositada no Field Museum (F). A coleção Sellow B 1452, traz o rótulo "Ex Museo Botanico Berolinense", com outra etiqueta gravada "Reliquiae d. Sello.". Entre as coleções de Sellow examinadas, de L. lasiocalycina, esta inclui as características encontradas na descrição original, como as medidas dos internós e inflorescências maduras e em frutificação, sendo indicada para lectotipificar a espécie.

O holótipo de L. subracemosa, depositado no herbário de Berlim (B), foi destruído durante a Segunda Guerra Mundial e um isótipo desta coleção Luetzelburg 710, foi encontrado no herbário de Munique (M), o qual foi designado para lectotipificar a espécie.

O exame das coleções tipo de Lippia lasiocalycina e Lippia subracemosa indicaram que em nada diferem, sendo proposto aqui o novo sinônimo.

14. Lippia pumila Cham., Linnaea 7: 218. 1832. Tipo. BRASIL. F.Sellow s.n .(holótipo, W!; isótipos, G!, HAL!, K!, P!, foto Field Museum!). Lippia nana Schauer in DC., Prodr. 11: 582. 1847. Tipo. BRASIL. Goiás: Serra do Urubu, J.B.E. Pohl 156 (holótipo, W!; isótipo K!), syn. nov.

A descrição de Lippia nana se sobrepõe à de Lippia pumila. A análise da coleção-tipo desses nomes deixa clara a sinonímia aqui proposta, uma vez que ambas apresentam xilopódio desenvolvido, lâmina foliar oval-lanceolada, inflorescências hemisféricas e indumento viscoso em toda a planta, além da presença de flores amarelas.

15. Lippia renifolia Turcz., Bull. Soc. Imp. Natulralistes Moscou 36(2): 204. 1863. Tipo: BRASIL. Goiás: 1841, G.Gardner 4336 (holótipo, $\mathrm{KW}$; fotografía, SI!; isótipos, G!, K OXF!, P!, W!). Lippia marrubiifolia Reichardt, Verh. Zool.-Bot. Ges. Wien 33: 322. 1884. Tipo: BRASIL. Minas Gerais, Paracatu, A. Varnhagen s.n. (holótipo, W!), syn.nov.

O exame dos isótipos de Lippia renifolia e da coleção-tipo de L. marrubiifolia indicam a proposta de sinonimização destas espécies por se tratarem de táxons idênticos, apresentando como características as folhas sésseis com lâminas reniformes. Lippia renifolia é uma espécie com distribuição nos cerrados e campos rupestres de Minas Gerais e Goiás, sendo muito característica pelas lâminas foliares sésseis, coriáceas, reniformes até orbiculares e inflorescências corimbiformes com brácteas membranáceas de coloração rosa.

16. Lippia rotundifolia Cham., Linnaea 7 : 230. 1832. Tipo: BRASIL: F.Sellow s.n. B1440 (lectótipo K!, aqui designado; isolectótipos, NY!, VT!, SI!).

A coleção Sellow s.n., depositada em K, com a numeração $\mathrm{B} 1440$, traz no rótulo a anotação de Chamisso ao descrever uma espécie nova e foi designada para lectotipificar este epíteto, por apresentar todas as características incluídas na descrição original. 
17. Stachytarpheta spathulata Moldenke, Phytologia 29(2): 76. 1974. Tipo: BRASIL. Minas Gerais: Rodovia Diamantina - Curvelo, $18 \mathrm{~km}$ by road southwest of Diamantina, Serra do Espinhaço, 10.IV.1973, W. R. Anderson 8515 (holótipo, TEX!).

Bouchea chascanoides Moldenke, Phytologia 3(2): 58. 1949. Tipo: BRASIL. Minas Gerais: Grão Mogol, Córrego dos Mortos, 11.XI. 1938, F. Markgraf, Melo Barreto \& A.C.Brade 3399 (holótipo, NY!; isótipo RB!), syn. nov.

Stachytarpheta spathulata subsp. mogolensis S. Atkins, Kew Bull. 60: 195. 2005. Tipo: BRASIL. Minas Gerais, Grão Mogol, 21.V.1982, M. C. H. Mamede et al. in CFCR 3388 (holótipo, SPF 23593!, isótipo, K!), syn. nov.

Moldenke (1949) descreveu Bouchea chascanoides com as mesmas características morfológicas presentes na descrição de Stachytarpheta spathulata, sendo as folhas espatuladas, a principal característica para reconhecimento desta última espécie. Além disso, na descrição original de $B$. chascanoides não há referência ao androceu, característica que permite separar Bouchea, que apresenta quatro estames perfeitos, de Stachytarpheta, com dois estames perfeitos e dois estaminódios. Desde a publicação de $B$. chascanoides, nenhum outro material havia sido coletado além da coleção-tipo e este táxon está ausente na flora de Verbenaceae de Grão Mogol, apesar do intenso trabalho de campo para o levantamento florístico na região (Salimena \& Silva 2009). O exame das coleçõestipo de $B$. chascanoides permitiu a identificação de dois estames funcionais e dois estaminódios, o que deixa clara a identidade desta espécie no gênero Stachytarpheta, aliada à presença de uma só bráctea floral e cálice não acrescente no fruto, características presentes neste gênero e ausentes em Bouchea. Com base nessas observações, B. chascanoides é aqui sinonimizada sob $S$. spathulata, pela ausência de caráter distintivo entre os dois táxons. Por outro lado, Stachytarpheta spathulata subsp. mogolensis, proposta por Atkins (2004), não apresenta sustentação taxonômica, uma vez que o caráter relacionado à morfologia da lâmina foliar, espatulada, descrita para a variedade-tipo x flabeliforme, descrita para $S$. spathulata subsp. mogolensis, é altamente variável nos espécimes analisados em herbários e em populações observadas em campo. Além disso, o comprimento da inflorescência, de $1-4 \mathrm{~cm}$ descrito para S. spathulata subsp. mogolensis está incluído nos limites de Stachytarpheta spathulata, como observado nas diversas coleções examinadas e também em campo. Desta forma, Stachytarpheta spathulata subsp. mogolensis é considerada um sinônimo de S. spathulata.

\section{Agradecimentos}

À Myndel Botanica Foundation e ao CNPq, Programas PROSUL e REFLORA, o apoio para revisão das coleções-tipo nos herbários $\mathrm{B}, \mathrm{BR}, \mathrm{G}$, NY, K, P, TEX e W outorgado à primeira autora. Aos curadores dos herbários visitados, a gentileza e permissão das visitas. Ao Dr. Jefferson Prado, o auxílio nomenclatural, ao Dr. Ricardo Secco, do Museu Goeldi, e ao revisor anônimo, as valiosas contribuições ao texto.

\section{Referências}

Atkins, S. 2004. Verbenaceae. In: Kadereit, J. W. (ed.). The families and genera of flowering plants. Vol. 7. Springer-Verlag, Berlin. Pp. 449-468.

Cantino, P.D.; Harley, R.M. \& Wagstaff, S.J. 1992. Genera of Labiatae: status and classification. In: Harley, R.M. \& Reynolds,T. (ed.). Advances in Labiatae Science. Royal Botanic Gardens, Kew. Pp. 511-522.

Chodat, R.H. 1902. Verbenacées. Plantae Hasslerianae. Bulletin de l'Herbier Boissier (ser. 2) 2: 820.

Jørgensen, P.M.; Nee, M.H. \& Beck, S.G. (eds.) 2014. Catálogo de las plantas vasculares de Bolivia. Monographs in Systematic Botany from the Missouri Botanical Garden 127(1-2): i-viii, 1-1744.

Marx, H.; O’Leary, N.; Yuan, Y.; Lu-Irving, P.; Tank, D.; Múlgura, M.E. \& Olmstead, R. 2010. A molecular phylogeny and classification of Verbenaceae. American Journal of Botany 97: 1647-1663.

Moldenke, H.N. 1965. Materials toward a monograph of the genus Lippia I. Phytologia 12: 174.

Moldenke, H.N. 1973. Verbenaceae. In: Woodson Jr., R.E \& Schery, R.W. (eds.). Flora of Panama. Annals of the Missouri Botanical Garden 60: 41-148.

Moldenke, H.N. 1981. Notes on new and noteworthy plants. CXLVI. Phytologia 48:290.

Múlgura de Romero, M.E.; Martínez, S. \& Suyama, A. 1998. Morfología de las inflorescencias en Lippia. (Verbenaceae). Darwiniana 36: 1-12.

Múlgura de Romero, M.E.; Martínez, S.; Atkins, S. \& Rotman, A.D. 2002. Morfología de las Inflorescencias en Verbenaceae, Verbenoideae III: Tribu Lantaneae p.p. Darwiniana 40: 1-15.

Salimena, F.R.G. 2002. Novos sinônimos e tipificações em Lippia sect. Rhodolippia (Verbenaceae). Darwiniana (1-4):121-125. 
Salimena, F.R.G. Stachytarpheta. In: Lista de espécies da flora do Brasil. Jardim Botânico do Rio de Janeiro. Disponível em $<$ http://floradobrasil.jbrj. gov.br/jabot/floradobrasil/FB15189>. Acesso em 23 setembro 2014.

Salimena, F.; França, F. \& Silva, T.R.S. 2009. Verbenaceae. In: Giulietti, A.M.; Rapini, A.; Andrade, M.J.G.; Queiroz, L.P. \& Silva, J.M.C. (orgs.). Plantas Raras do Brasil. Conservação Internacional. Belo Horizonte, Minas Gerais. Pp. 399-405.

Salimena, F.R.G.; Kutschenco, D.C.; Monteiro, N.P. \& Myssen, C. 2013. Verbenaceae. In: Martinelli,G. \& Moraes, M.A. (orgs.). Livro Vermelho da Flora do Brasil. CNCFLORA. Instituto de Pesquisas Jardim Botânico do Rio de Janeiro, Rio de Janeiro. Pp. 1010-1016.

Salimena, F.R.G. \& Múlgura, M. 2014. Lippia. In: Lista de espécies da flora do Brasil. Jardim Botânico do Rio de Janeiro. Disponível em $<$ http://floradobrasil. jbrj.gov.br/jabot/floradobrasil/FB15170> Acesso em 03 agosto 2014.

Salimena, F.R.G. \& Silva, T.R.S. 2009. Flora de GrãoMogol, Minas Gerais: Verbenaceae. Boletim de Botânica da Universidade de São Paulo 27: 119-126.

Salimena, F.R.G.; Thode, V.; Mulgura, M.; O’Leary, N.; França, F.; Silva, T.R.S. \& Souza, V.C. Verbenaceae. In: Lista de espécies da flora do Brasil. Jardim Botânico do Rio de Janeiro. Disponível em $<$ http:// floradobrasil.jbrj.gov.br/jabot/floradobrasil/ FB246> Acesso em 23 setembro 2014.

Silva, T. R. S. 2001. Lectotypifications and neotypification in Lantana L. and Lippia L. (Verbenaceae). Taxon 50: 1115-1118.

Silva, T.R.S. \& Salimena, F.R.G. 2002. Novas combinações e novos sinônimos em Lippia e Lantana (Verbenaceae). Darwiniana 40 (1-4):57-59.

Silva, T.R.S. \& Salimena, F.R.G. 2014. Lantana. In: Lista de espécies da flora do Brasil. Jardim Botânico do Rio de Janeiro. Disponível em $<$ http:// floradobrasil.jbrj.gov.br/jabot/floradobrasil/ FB15163> Acesso em 03 agosto 2014.

Thiers, B.[continuously updated]. Index Herbariorum: a global directory of public herbaria and associated staff. New York Botanical Garden's Virtual Herbarium. Disponível em $<$ http://sweetgum.nybg. org/ih>. Acesso em 11 junho 2014. 